\title{
Visual problems in Parkinson's disease: A questionnaire survey
}

\author{
Sally-Ann McDowell and John P. Harris
}

\author{
Department of Psychology, The University of Reading, Earley Gate, Whiteknights, \\ Reading RG6 6AL, UK \\ Correspondence to: John P. Harris at above address
}

\begin{abstract}
A questionnaire was completed by 53 putative sufferers from Parkinson's disease and 31 putative age-matched normal controls. The aim of the questionnaire was to elicit reports of any changes in visual perception. The incidence of self-reported Parkinsonian symptoms was very much higher in the patient group than in the controls. The patients reported significantly more problems with depth and motion perception than the controls. They also reported a significantly higher incidence of hallucinations, double vision and the need to turn the head to see objects in the periphery. However, the reported incidence of changes in brightness, colour, shape and size perception was not significantly different in the two groups. The results are discussed with reference to laboratory studies of Parkinsonian vision and to the likely neurological basis of some of the changes.
\end{abstract}

Keywords: Parkinson's disease - Questionnaires - Vision

\section{INTRODUCTION}

Parkinson's Disease (PD) was originally thought of as a motor disorder, in which "the senses and intellect are unimpaired" (Parkinson, 1817), but much recent work suggests that this is too narrow a description. Although the nature and extent of any intellectual impairments are still controversial (see, for example, Brown and Marsden, 1990), there is increasing evidence for changes in vision in PD. At the anatomical and physiological levels, it is known that retinal dopaminergic neurones (Nygen-Legros and Savy, 1988) and the latency of the Visual Evoked Potential (Bodis-Wollner and Yahr, 1978) are abnormal. Behaviourally, these changes may correspond to elevated contrast thresholds for medium and high spatial frequencies (Bodis-Wollner, 1988) and a reduction of their perceived contrast in peripheral vision (Harris et al., 1992). In addition, it is known that eye movements may be abnormal in PD, so that saccade latency may be increased and saccade amplitude reduced (Cogan, 1964; Corin et al., 1974; Bronstein and Kennard, 1984). Neurological evidence for problems with more cognitive aspects of vision is less compelling but, if the disorder involves a generalised hypoactivity of dopamine systems (Barbeau et al., 1975), abnormalities of occipital, parietal and frontal cortex are likely, since dopamine is found in those areas in primates (Berger et al., 1991).

The present study had two aims. The first aim was to investigate whether some of the visual changes found in some laboratory studies have been noticed by patients outside the laboratory (and so are likely to be of both clinical and practical significance). The second aim was to obtain an indication of whether other types of visual abnormality, not previously described in the literature, might occur in a significant proportion of patients. Should this be the case, more refined methods could be employed in further investigations.

From existing laboratory studies on Parkinsonian patients, one might expect oculomotor changes, changes in sensitivity to contrast, motion sensitivity deficits, reduced sensitivity in peripheral vision, problems in visual orientation, visual neglect, problems in visual search and visual attention, and impairment of some visuospatial abilities, though not necessarily in all patients. However, there is very little evidence in the literature that PD patients experience any subjective changes in vision. A previous study (Phillipson and Harris, 1985) investigated perceptual changes in schizophrenia with a questionnaire. It was decided to use a similar methodology to investigate possible perceptual 
changes in PD. The questionnaire was designed to allow it to be completed by both patients with PD and individuals with no known neurological impairment. The approach was essentially exploratory, involving the investigation of areas of visual experience which might be abnormal for several reasons, suggesting more detailed investigation in future studies.

\section{METHOD}

\section{The questionnaire}

The questionnaire was a typed A4 booklet. The first page contained information about the general nature of the study, what was required of the respondent, and the names and contact telephone numbers of the two investigators (the authors). The first section of the booklet was used to record details of age and sex of respondents. The existence of chronic diseases, including year of diagnosis, and use of medication, including dosage, were recorded. Tables were provided with space to report details of up to three illnesses and five drugs, if necessary. Other questions in this section referred mainly to PD symptoms and possible antiParkinsonian medication, but they were designed in such a way that a non-sufferer could answer them (e.g. 'Do any of your limbs feel rigid?'). This section also asked about the possible occurrence of tremor and akinesia, difficulties with walking through doorways, and 'freezing' episodes. Respondents were also asked about the occurrence of fainting, migraine or motion sickness. These questions were designed to identify and exclude from the analysis at least some of the visual effects due to these problems.

The second section of the questionnaire was used to record information about changes in vision, such as changes in colour ('Do you think you see colours in the same way as you used to?'); brightness ('Do objects look as bright to you as they used to?'); depth ('Can you judge depth as well as you used to? - by depth, we mean, for example, when looking at two objects, can you determine which is closer to you?'); shape; size ('Do any everyday objects appear to be different in shape/size from what they used to be?'); and motion ('Do people or vehicles on the street appear to move differently from how they used to?'). There were also questions about double vision ('Do you ever get double vision?'); visual hallucinations (see later discussion); and the need to turn the head more often to see objects ('Do you find that you need to turn your head to look at objects at the side of you more than you used to?'). Respondents were also asked about other visual problems and encouraged to describe them in detail. The wording of some questions was altered in an attempt to guard against acquiescence bias. Thus, some questions were phrased to ask whether some aspect of vision was the same as it used to be, while other questions asked if some aspect of vision was different. The subject's response options were either Usually (or Often)/Sometimes/Never or Yes/No.

Approval for the study had been obtained from the University of Reading Ethics and Research Committee and the West Berkshire Health Authority Ethics and Research Committee.

\section{Subjects}

Two groups of subjects took part in the study. One group was composed of putative sufferers from PD and the other was made up of individuals over the age of 40 with no reported neurological or ophthalmological impairment. Respondents were recruited by advertising within the University of Reading, through local branches of the UK Parkinson's Disease Society, and at a conference for younger sufferers from PD. All subjects, whether Parkinsonian or control, who visited our laboratory to take part in other experiments were approached to take part in this study. About 400 questionnaires were issued, of which 99 were completed and returned. It was not possible to calculate an exact response rate because many questionnaires were issued to local organisers and it has not been possible to determine how many were passed on to potential respondents. A major limitation of the study is the reliance on self-report for the diagnosis of PD in the patient group (which is incorrect in about $25 \%$ of cases, even when made by a neurologist), and the absence of neurological or other illness in the control group. Another major limitation is that the normal and Parkinsonian samples were essentially self-selected and this might have biased the completion of questionnaires towards those with visual problems.

It was found that the patient $(n=62$, of whom 31 were male) and control $(n=37$, of whom 17 were male) groups did not match in age, but this could be remedied by excluding all questionnaires from subjects aged over 75 years $(\mathrm{PD}=6$, controls $=2)$. Additionally, all subjects who reported frequent migraines $(\mathrm{PD}=2$, controls $=2$ ) and frequent episodes of motion sickness $(\mathrm{PD}=1$, controls $=2)$ were excluded. Frequent fainting episodes were not reported by any subjects. This left 31 questionnaires from controls (15 of whom were male) and 53 questionnaires from PD sufferers (28 of whom were male). The control group had a mean age of 55.10 years $(\mathrm{SE}=1.60$ years) and the Parkinsonian group had a mean age of 57.80 years ( $\mathrm{SE}=1.08$ years). The two groups did not differ significantly in age $(\mathrm{t}=1.45, \mathrm{df}=82, p>0.05)$. 
Statistical Analysis

Partly to simplify analysis, and partly because self-ratings of symptom frequency may not be valid, the data were dichotomised into 'problem present' (Often and Sometimes responses) and 'problem absent' (Never responses). Chi-squared tests were employed to test the significance of differences in frequency of symptoms in PD and control subjects. This statistic was also employed to test the responses to each of the perceptual questions in PD and control subjects.

\section{RESULTS}

\section{Frequency of Parkinsonian symptoms}

The percentages of each group who reported the presence of symptoms or perceptual problems can be found in Table I. The four PD symptoms (tremor, rigidity, freezing, difficulty at doorways) occurred more often in the PD group than the control group. Only $3 \%$ of the controls reported rigidity, freezing or difficulties in negotiating doorways, whereas $89 \%$, $60 \%$ and $56 \%$ of the PD group reported these problems, respectively. The incidence of tremor was $16 \%$ in the control group and $85 \%$ in the PD group. The apparently high incidence of this Parkinsonian symptom in the control group may reflect the wording of the question ('Do you experience tremor or trembling in any of your limbs?'). Although the term 'trembling' was intended to explain or amplify the word 'tremor', it may have led to the reporting of minor symptoms which would not have been classified as 'tremor' by a clinician. However, these results suggest that the selfreport technique employed in this study discriminated the two groups rather well. One of the aims of the study was to investigate whether specific visual changes were associated with particular Parkinsonian motor symptoms. Although there were trends in the data, none of these reached statistical significance, so they will not be pursued further in this paper.

\section{Perceptual differences between patient and control groups}

Changes in the perception of colour were rare (less than $10 \%$ in both groups). Changes in the brightness of objects were reported by $15 \%$ of the PD group but by only $3 \%$ of the control group. Less than $25 \%$ of both groups reported changes in the shape of objects and less than $20 \%$ reported changes in the size of objects. None of the differences in these variables between the groups was significant. Given the caveats about sampling mentioned earlier, the absence of differences between the groups are important when considering the interpretation of differences on other questions.

Significant differences in frequency were found for reported changes in the ability to judge depth, in motion perception, in the occurrence of double vision, in the frequency of visual hallucinations, and in the need to turn the head to the side to see objects in the periphery. Less than $10 \%$ of the control group reported problems with judging depth, whereas over $25 \%$ of the PD group had problems. Almost $20 \%$ of the PD group reported problems in motion perception, whereas less than $5 \%$ of the control group did so. Double vision was reported by over $50 \%$ of the PD group (mainly when reading or watching television) whereas only $10 \%$ of the control group ever experienced double vision. Visual hallucinations were reported by $43 \%$ of the PD group, $45 \%$ of whom

TABLE I: Percentage of subjects reporting the presence of problems in perception or the presence of a symptom

\begin{tabular}{lcccc}
\hline Symptom/problem & $\begin{array}{c}\text { \% of PD group } \\
\text { reporting } \\
\text { problem }\end{array}$ & $\begin{array}{c}\text { \% of control } \\
\text { group reporting } \\
\text { problem }\end{array}$ & $\chi^{2}$ & $p$ \\
\hline Tremor & 85 & 16 & 38.4 & $<0.001$ \\
Rigidity & 89 & 3 & 58.3 & $<0.001$ \\
Freezing & 60 & 3 & 26.8 & $<0.001$ \\
Difficulty at doorways & 56 & 3 & 5.1 & $<0.05$ \\
Depth perception & 26 & 6 & 4.2 & $<0.05$ \\
Motion perception & 19 & 3 & 7.3 & $<0.01$ \\
Need to turn head to side & 43 & 14 & 18.1 & $<0.001$ \\
$\quad$ to see peripheral objects & & & 7.2 & $<0.01$ \\
Double vision & 57 & 10 & 0.3 & $>0.05$ \\
Hallucinations & 43 & 14 & 3.0 & $>0.05$ \\
Colour perception & 4 & 6 & 0.3 & $>0.05$ \\
Brightness perception & 15 & 16 & 0.3 & $>0.05$ \\
Shape perception & 21 & 13 & & \\
Size perception & 17 & & & \\
\hline
\end{tabular}


accounted for them as effects of the medication they were taking. Surprisingly, almost $15 \%$ of the control group also reported visual hallucinations, although this was still significantly less than the PD group. This reported incidence may reflect question wording ('Do you ever have visual hallucinations? i.e. see strange flashes of light, shadows, or things which are not there'). Some controls might have replied positively to the question because they had experienced, for example, phosphenes produced by pressure on the eye from the pillow as they turned over in bed at night. More than $40 \%$ of the PD group reported having to turn their head more often to see objects in the periphery in comparison with less than $15 \%$ in the control group.

\section{Qualitative descriptions of symptoms by patients}

Whereas the control group reported only long- or short-sightedness under 'other problems in vision', the PD group reported many different problems. Many had problems focusing, with the problem becoming worse when the effects of medication were wearing off. Several sufferers noted that vision was better after they had been awake for several hours and the eyes had had time (as they put it) to 'warm up'. Tunnel vision was reported by one sufferer. Another sufferer, who reported tremor as a PD symptom, described tremor in the eyes as well as the limbs. Several of the PD group experienced pain in the eyes which may have resulted from dry eyes. Two sufferers reported that they often needed more light to see clearly, that vision was worse in the dark, and that they experienced a general blurring of vision. Three respondents commented on visual effects of the medication they were taking. Pergolide was reported to make dreams more colourful, benzhexol to contribute to blurring of vision and also to 'make the eye muscles relax', which presumably also contributed to blurring.

\section{DISCUSSION}

This questionnaire study has suggested that our sample of putative PD sufferers are aware of a range of problems with their vision which are not experienced by the control subjects to the same extent. Apart from anecdotal comments, no other study, to our knowledge, has explored this idea. Although our classification of subjects depended on self-report, answers to other questions suggested that the incidence of possible Parkinsonian symptoms was very low in the control group, and that the reported visual changes were unlikely to be due to migraine, fainting or motion sickness.
No differences in colour, shape and size perception were found between the two groups. Alterations in visuospatial ability have been commented upon in PD (see, for example, Brown and Marsden, 1985) and it might have been expected that PD sufferers would report changes in size and/or shape perception, but this was not so. No difference in reports of changes in brightness perception was found. Such a difference might have been expected, since the dopaminergic amacrine and interplexiform cells of the retina are involved in the process of dark adaption (Ehinger, 1983). Their hypo-activity in PD probably results in a retina which is too dark-adapted for the prevailing luminance (Beaumont et al., 1987). A characteristic of dark adaption is a reduction in apparent luminance contrast.

This study found potentially important differences between PD sufferers and age-matched controls in the occurrence of several reported changes in visual perception. One difference is in the reported ability to judge depth, which might reflect problems with visuospatial processing. Lee et al. (in press) found abnormalities in PD in the three-dimensional mental rotation of wire-frame figures. This evidence for abnormality in the internal representation of threedimensional space is consistent with the present finding that many PD patients report that they find it more difficult to judge depth.

A second difference observed between the groups was in reported abnormalities in the way in which people and vehicles appear to move on the street (as suggested to us earlier by several sufferers). This reported problem in motion perception is in agreement with psychophysical findings from Haug et al. (1994) and Mestre et al. (1990). Haug et al. measured motion thresholds and found that PD subjects had higher thresholds to low contrast (3-7\% achromatic contrast), small displacement stimuli. As defects only occurred at low contrast, these authors believe that only the magnocellular pathway is affected. Mestre $e t$ al. claim that all visual deficiencies in PD are related to loss of motion sensitivity. However, Bodis-Wollner (1988) and McDowell (1995) have reported losses in foveal contrast sensitivity for stationary gratings in PD.

The PD group were significantly more likely to report double vision than the controls. This may reflect the effects of the disorder on fine oculomotor control (see, for example, Cogan, 1964; Corin et al., 1975; Bronstein and Kennard, 1984).

Previous research suggests that PD subjects find items in the periphery more difficult to see than agematched controls (see, for example, Harris et al., 1992; McDowell, 1995). The present study found that more 
PD subjects needed to turn their heads to the side to see objects in the periphery which could be a direct consequence of a deficit in peripheral vision. In fact, one PD subject reported 'tunnel vision' and it is possible that this deficit in peripheral vision could be a mild form of tunnel vision (as suggested by Harris et al., 1992).

\section{CONCLUSION}

The results of this study indicate that PD sufferers do seem to be aware of changes in vision which are not experienced (or, at least, not reported) by the normally ageing population. These include, especially, selfreported problems with motion perception, depth perception, peripheral vision and double vision. Some sufferers account for the changes as being due simply to ageing, though the present study has found this not to be the case. Several problems appear to be effects of Parkinson's disease itself. The study suggests that some visual effects which have been found in laboratory studies may be significant enough in some patients to be of clinical and practical importance and that there are other visual problems in Parkinson's disease which may merit investigation with more precise methods.

\section{Acknowledgements}

We thank the MRC for a Research Studentship (S-AMcD), the Wellcome Trust and the Parkinson's Disease Society for project grants (JPH), and all our respondents for their help and interest.

\section{REFERENCES}

Barbeau A, Campanella G, Butterworth RF and Yamada $\mathrm{K}$ (1975) Uptake and efflux of 14C dopamine in platelets: evidence for a generalised defect in Parkinson's disease. Neurology, New York, 25, 1-9.

Beaumont SM, Harris JP, Leendertz JA and Phillipson OT (1987) The pupillary light reflex in mild Parkinson's disease. Clinical Vision Science, 2 (2), 123-129.

Berger B, Gaspar P and Verney C (1991) Dopaminergic innervation of the cerebral cortex: unexpected differences between rodents and primates. Trends in Neurosciences, 14, 21-27.

Bodis-Wollner I (1988) Altered spatio-temporal contrast vision in Parkinson's disease and MPTP-treated monkeys: the role of dopamine. In: Dopaminergic Mechanisms in Vision (Eds I Bodis-Wollner and M Piccolino), pp. 205-220. Alan R Liss, New York.
Bodis-Wollner I and Yahr MD (1978) Measurements of visual evoked potentials in Parkinson's disease. Brain, 101, 661-671.

Bronstein AM and Kennard C (1984) Predictive eye movements in normal subjects and in Parkinson's disease. In: Theoretical and Applied Aspects of Eye Movement Research (Eds AG Gale and F Johnston), pp. 463-472. Elsevier, Amsterdam.

Brown RG and Marsden CD (1985) Visuospatial function in Parkinson's disease. European Neuroscience Association on Clinical Neuropsychology Meeting at Zurich, Switzerland.

Brown RG and Marsden CD (1990) Cognitive function in Parkinson's disease: from description to theory. Trends in Neurosciences, 13 (1), 21-29.

Cogan DC (1994) Brain lesions and eye movements in man: In: The Oculomotor System (Ed MB Bender), pp. 417-423. Harper and Rowe, New York.

Corin MS, Elizan TS and Bender MB (1984) Oculomotor function in patients with Parkinson's disease. Journal of Neuroscience, 15, 251-265.

Ehinger B (1983) Functional role of dopamine in the retina. In: Progress in Retinal Research (Eds N Osbourne and G Chader). Pergamon Press, Oxford.

Harris JP, Calvert JE and Phillipson OT (1992) Processing of spatial contrast in peripheral vision in Parkinson's disease. Brain, 115, 1447-1457.

Haug BA, Trenkwalder C, Arden GB, Oertel WH and Paulus W (1994) Visual thresholds to low-contrast pattern displacement, color contrast, and luminance contrast stimuli in Parkinson's disease. Movement Disorders, 9 (5), 563-570.

I.ee AC, Harris JP and Calvert JE. Impairments of mental rotation in Parkinson's disease. Neuropsychologia (in press).

McDowell SA (1995) The Effects of Peripheral Vision on Motor Initiation in Parkinson's disease. Unpublished PhD. Thesis, The University of Reading.

Mestre D, Blin O, Serratrice G and Pailhous J (1990) Spatiotemporal contrast sensitivity differs in normal ageing and Parkinson's disease. Neurology, 40, 1710-1714.

Nguyen-Legros J and Savy C (1988) Dopamine innervation of the vertebrate retina: morphological studies. In: Dopaminergic Mechanism in Vision (Eds I Bodis-Wollner and M Piccolino), pp. 253-265. Alan R Liss, New York.

Parkinson J (1817) An Essay on the Shaking Palsy. London: Sherwood, Neely and Jones.

Phillipson OT and Harris JP (1985) Perceptual changes in schizophrenia: a questionnaire survey. Psychological Medicine, 15, 859-866.

(Received 10 November 1995; accepted as revised 31 January 1997) 


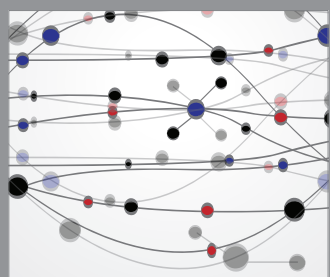

The Scientific World Journal
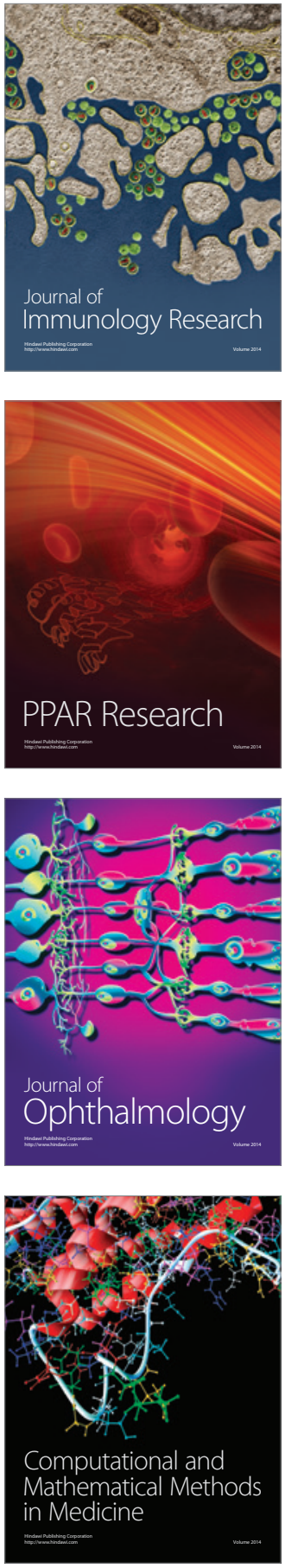

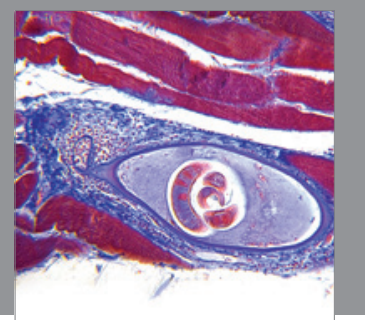

Gastroenterology

Research and Practice
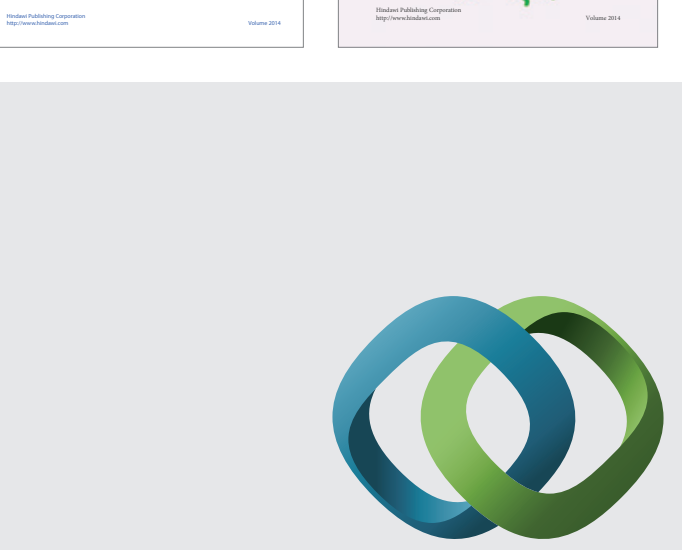

\section{Hindawi}

Submit your manuscripts at

http://www.hindawi.com
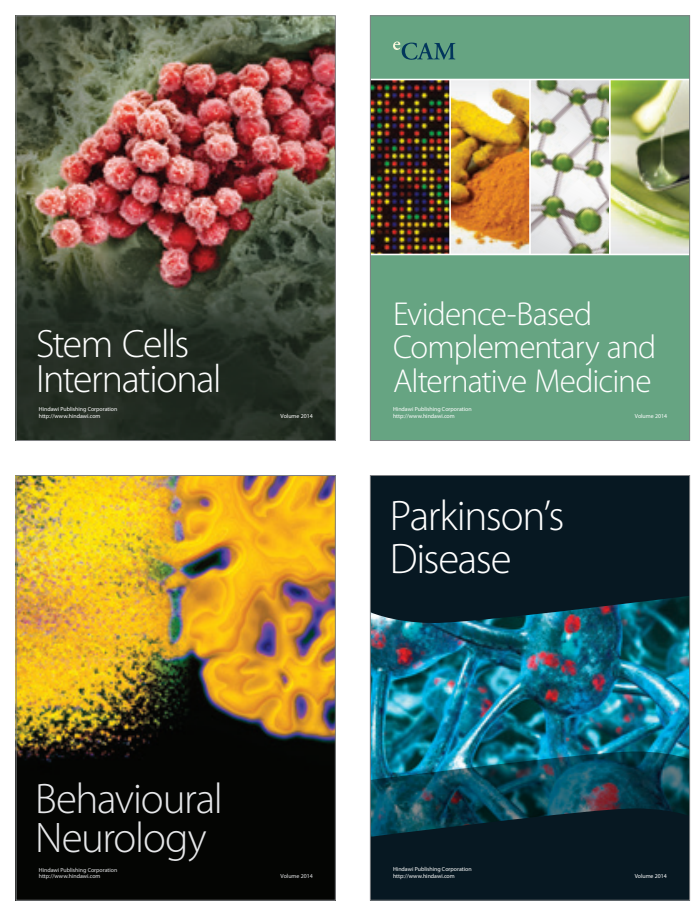

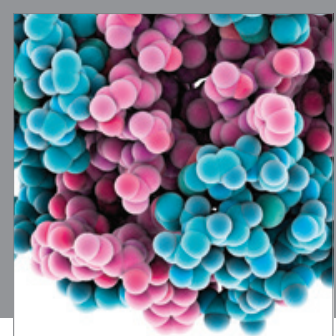

Journal of
Diabetes Research

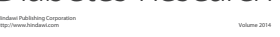

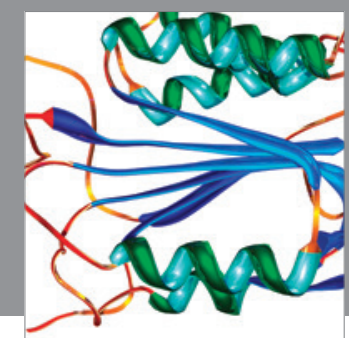

Disease Markers
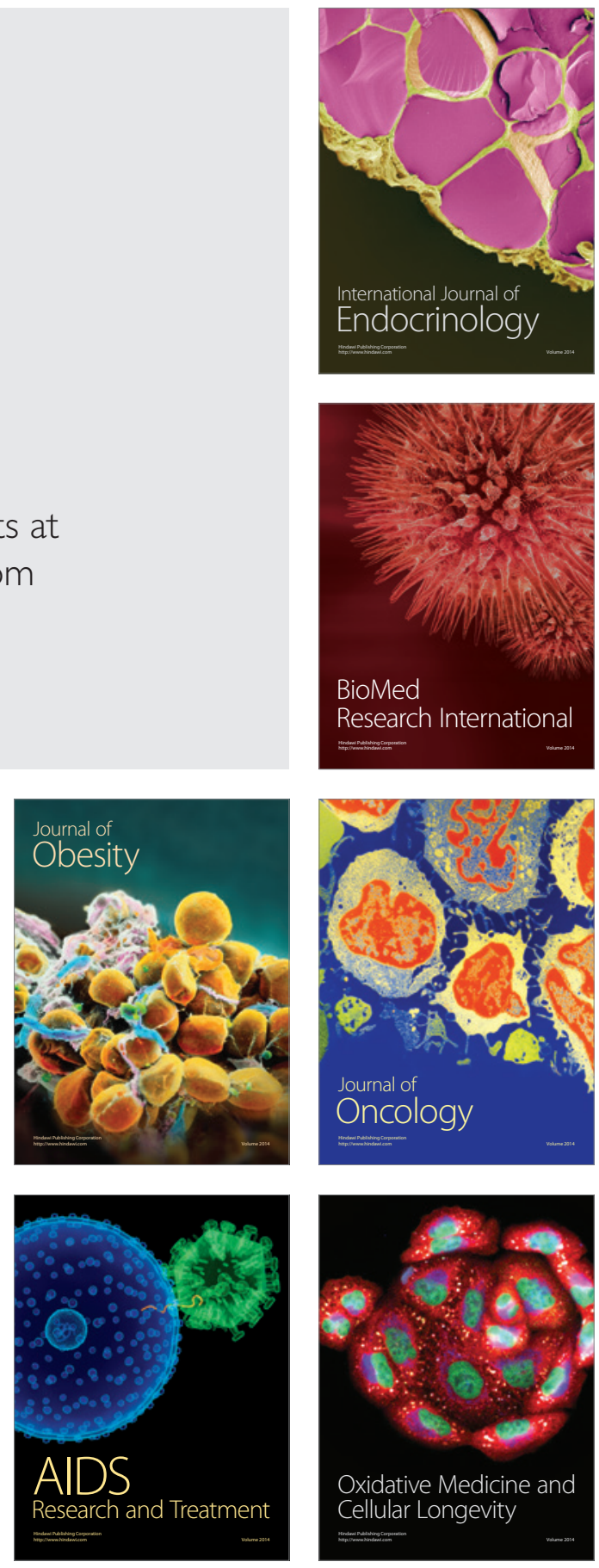